\title{
Accident Detection and Surveillance System using Wireless Technologies
}

\author{
Purva Javale ${ }^{1,}$ Shalmali Gadgil ${ }^{2,}$ Chinmay Bhargave ${ }^{3,}$ Yogesh Kharwandikar ${ }^{4}$, \\ Prof. Vaishali Nandedkar \\ (Information Technology, Pune University, India), \\ 2 (Information Technology, Pune University, India) \\ 3 (Information Technology, Pune University, India) \\ ${ }^{4}$ (Information Technology, Pune University, India)
}

\begin{abstract}
The symbiosis between communication technologies and vehicles offers opportunity to improve assistance to people injured in traffic accidents, by providing information about the accident to reduce the response time of emergency assistance services. Determining the required resources for accident could significantly reduce the number of casualties. This paper presents a system prototype especially designed to detect and provide faster assistance to traffic accident victims. The proposed system requires each vehicle to be endowed with a control unit $(C U)$ responsible for detecting accident and onboard unit $(O B U)$ reporting accident location for providing the necessary resources for the rescue operation.
\end{abstract}

Keywords: Control Unit, Emergency Assistance Service, Onboard Unit, Traffic Accidents,

\section{INTRODUCTION}

In the last few decades, the total number of vehicles around the world has experienced a remarkable growth. The effect of this situation is the increase of traffic accidents on the road, representing a serious problem in most countries.

In 2008, Road accidents ranked fourth among the leading causes of death in the world. Nearly 1.3 million people die every year due to road accidents and 20 to 50 million people suffer non-fatal injuries, with many sustaining disabilities as a result of their injury. According to the World Health Organization (WHO), road accidents are the sixth leading cause of deaths in India and socio-economic losses in the young and middleaged population. Road traffic injuries also place a huge burden on the health sector in terms of pre-hospital and acute care and rehabilitation (2001 to 2011). During the same period, the number of road accidents in the country increased by $2.1 \%$. Similarly, the number of road accident fatalities and the number of persons injured in road accidents in the country between 2001 and 2011 increased by $5.8 \%$ and $2.4 \%$.

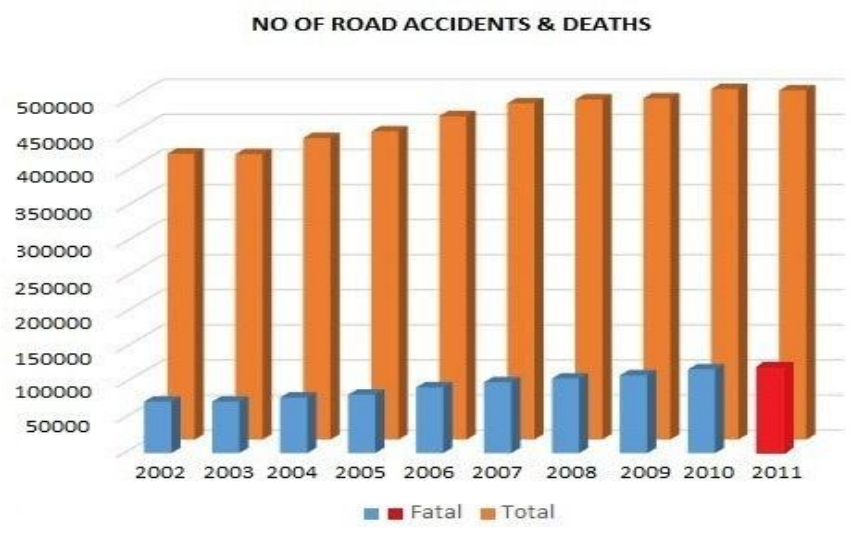

Figure 1: Number of Accidents \& Deaths

The proportion of fatal accidents in the total road accidents has consistently increased since 2002 from 18.1 to $24.4 \%$ in 2011 . The severity of road accidents measured in terms of persons killed per 100 accidents has also increased from 20.8 in 2002 to 28.6 in 2011 . Road accident cases in the country have marginally decreased by $0.02 \%$ during 2012 , while the casualties in road accidents in the country have increased by $1.3 \%$ during 2012 as compared to 2011. 
State wise percentage of accident deaths in 2012 is shown below:-

PERCENTAGE OF ACCIDENTAL DEATHS IN INDLA-2012

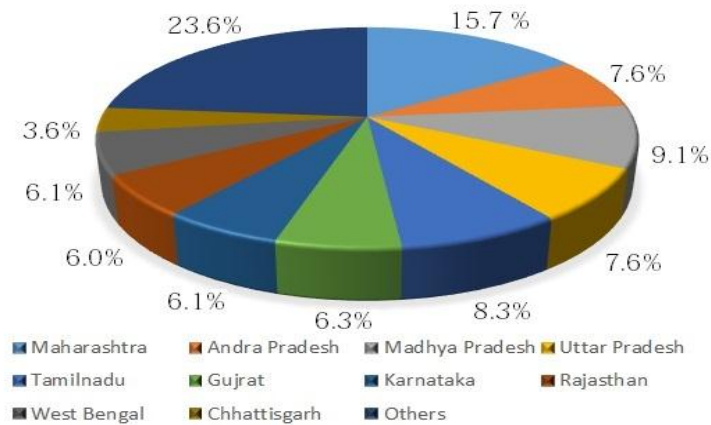

Figure 2: Percentage of Accidental Deaths in 2012

When a traffic accident takes place, assisting injured passengers as soon as possible is most important to minimize the negative effects on their health. According to research in 2011 out of 500000150000 passengers died due to lack of timely assistance.

Mortality from traffic accidents can be classified into three phases: First phase involves casualties in the first few minutes or seconds after an accident (about 10\% of all deaths). In Second phase, deaths occurred in few hours after the traumatic incident, has the highest mortality rate ( $75 \%$ of all deaths) and is the phase during which the highest death rate can be avoided by providing proper emergency assistance. In third Phase, $15 \%$ of the total mortality occurred, and takes a high amount of resources to reduce mortality. In this phase, mortality rate increases due to lack of timely emergency assistance.

For a noticeable reduction in assistance time, two major steps must be taken: 1) Detecting fast and accurate accident location and reporting it to nearest Emergency Service Providers 2) Quick and efficient evacuation of victims trapped inside a vehicle. Numerous efforts have been undertaken by automobile manufacturers to reduce road accidents. The initiatives have been managed to improve traffic safety, achieving a reasonable reduction of road deaths. However, accidents can still occur, and a quicker response from emergency services could significantly decrease the number of injuries and casualties as well as the impact of such accidents on traffic.

The proposed system is designed to improve the emergency assistance services, which offers automated detection using wireless communication technologies. This system does not focus on reducing the number of accidents but on improving emergency assistance with fast and efficient management of the available resources, thereby increasing the chances of survival for people injured in traffic accidents.

The system requires each vehicle to be endowed with a control unit $(\mathrm{CU})$ and an onboard unit $(\mathrm{OBU})$ to improve emergency assistance on the road. A Smartphone application is developed to provide the emergency services. The proposed application monitors the trigger to detect when an accident has occurred. Accident detection is followed by actions, such as sending accident details via SMS to the emergency services providers.

\section{Literature Review}

A number of research projects held by research institutes and car manufacturers around the world have been focusing on inter vehicle communication systems. Considering worldwide systems for emergency reporting regardless of their communication method whether it's wired or wireless, helped to define the strong objectives about our proposed systems. Some of the projects related to the proposed system are listed below:

Manuel Fogue and his partners [1] proposed a prototype architecture called e-NOTIFY, a novel proposal designed to improve the chances of survival for passengers involved in car accidents. The proposed system offers automated detection, reports, and assistance to passengers involved in road accidents by exploiting the capabilities offered by vehicular communication technologies. The goal of this system is to provide an architecture that allows 1) direct vehicle to vehicle (V2V) involved in the accident, 2) automatic sending of a data file containing important information about the incident to the $\mathrm{CU}$, and 3) a preliminary and automatic assessment of the damage to the vehicle and its occupants, based on the information received from the involved vehicles, and a database of accident reports. According to the reported information and the preliminary accident estimation, the system will alert the required rescue resources to optimize accident assistance.

Tanushree Dalai [2] proposed a Telematic model which has three main modules. The system is intended to capture the location of the vehicle through GPS receiver, send the location information to vehicle owners mobile number through SMS and also to the Telematics operator server through GPRS. It consist of modules which are 1) A GPS receiver is required to get the accurate information from the GPS satellites 
GSM/GPRS 2) The GSM/GPRS modem utilizes the GSM network to send the location of the accident and other necessary information. The modem can be controlled by a microcontroller through AT Command set. 3) The microcontroller unit (MCU) receives data from the GPS, processes all data, send location of the vehicle to server and vehicle owner/family members.

In E. Davila, eCall [3] the authors present an automatic emergency alert system for two-wheeled. This system has features like crash detector and an eCall box, which can be connected over a wired or wireless link which was developed only for two wheelers. The box provides the notification (emergency call) service, which sends eCall's Minimum Set of Data to the Public Safety Answering Point. Early experimental results showed systems reliability of the detector-box wireless communication, and detection of frontal, lateral and roll-over crash types. This system alerts the driver of the vehicle about accidents on the coming roads and also advices to control the speed i.e. acceleration and braking.

Richard Bossom and his partners [4] proposed a prototype COMeSafety, Its main goal is to develop a European set of standards to support wide implementation and deployment of cooperative Intelligent Transportation Systems (ITS). Also it aims at coordinating the activities toward the realization of cooperative systems on European roads, focusing on all issues related to Vehicle to vehicle (V2V) and Vehicle to Infrastructure (V2I) communications. Such technologies do not prevail in India hence we proposed this system.

Ruth Silverman [5] and others have presented paper on optimal algorithm for searching. They have mentioned few algorithms such as

- The BBD-Tree

- Great Circle Distance Formula

- Approximate k-Nearest Neighbors

\subsection{Great Circle Distance Formula}

There are several ways to define a great circle. One of the most useful in understanding its properties is to look at the intersection of a plane and a sphere. This will always be a circle, but usually not a great circle. As an example, consider the paths between Portland Oregon and Portland Maine. Both are at about 45 degrees north. Look at the plane that is perpendicular to the z-axis and intersects the earth at $45 \mathrm{~N}$.

The circle that the plane makes is called a small circle. It passes through both cities, but is not the shortest path. It is at the same latitude everywhere. (Because in this example we assume both cities are on the same latitude, this is also a thumb line at azimuth of 90 degrees.)

One definition of a great circle is the intersection of a plane and a sphere where the origin of the sphere is on the plane. Thus you can tilt the plane about the line that goes through the two cities until the plane passes through the center of the earth. This procedure works to connect any two points on a spherical earth with a great circle. The correct plane is unique except for two points directly opposite each other as seen from the earth center. These are called antipodal points. The North and South Poles are one example of an antipodal pair of points.

The resulting intersection from this procedure is now a great circle. (Mathematically you can take the three points of the end points and the earth center and define this plane) The great circle is the shortest distance between the two points along the surface of the spherical earth. On the real earth this method produces a path adequate for navigation. It is not the absolute shortest path though [6][7]

After studying the existing systems, we designed and developed a new Prototype, which works on same principal of informing accident to control room.

Infrastructure implementation cost is extremely high for existing systems. This prototype is an optimal alternative for these systems. Systems like eCall [3], eNotify[1] are ideal but in India due to inadequate resources and dense population ,these systems are difficult to implement.

The proposed system not only detect accident but also make provisions for emergency assistance services. This system provide fully automatic solution for accident detection and emergency assistance provision. In addition to above features, proposed prototype provide the facility of informing accident information to accident victim's family.

\section{Accident Detection And Surveillance System}

The proposed application is developed for the Android-based smart phones. In particular, the system relies on the microcontroller to sense accident event and GPS technology to find the exact location of the accident. With the use of Bluetooth Module, eliminates the need for physical connection, making it more robust against hardware damages. Since a data communications channel between the smart phone and server is required, it can be established using TCP/IP. Mobile telephony services such as SMS are used. The system is configured to send an SMS about accident information, such as current GPS co-ordinates of accident location, to Emergency Service Providers. 


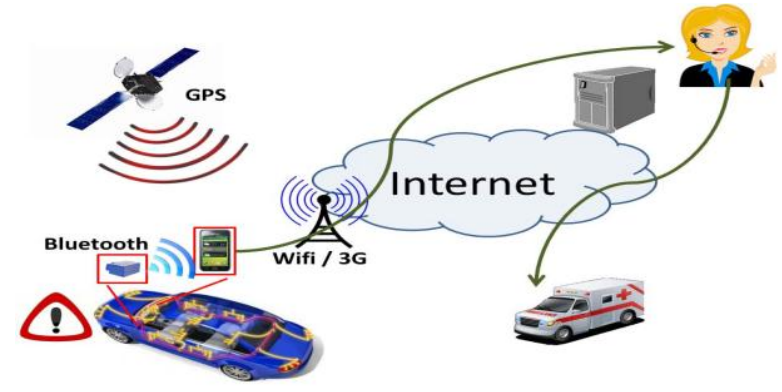

Figure 3: General System

It is not feasible to test this system by actually crashing the car. Instead, relay can be used to simulate the accident event. Fully compressed relay can be considered as accident condition.

On sensing the collision, Microcontroller sends a signal triggering an Accident event. This signal is then send via Bluetooth. Then accident location (longitude and latitude) is send to server through smart phone via TCP/IP. The server then search for the nearest hospitals/police \& victim's family members and calculate their distance from accident location. A list is created according to minimum distance, to provide the emergency assistance as soon as possible.

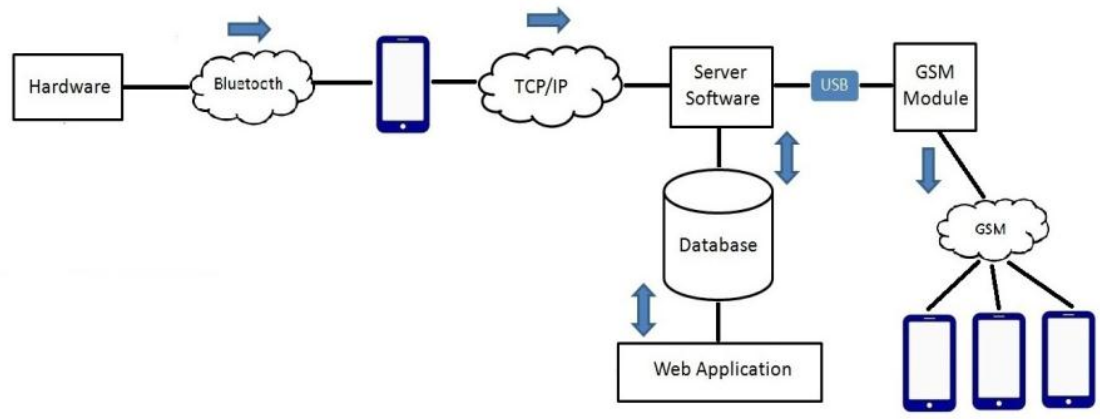

Figure 4: Architecture of Accident Detection and Surveillance System

\subsection{Accident Detection Algorithm:}

1. Waiting for detection of Collision

2. Informing Smartphone about Accident via Bluetooth.

3. After receiving accident information, get coordinates of current location.

4. Providing the location coordinates to server for further processing

5. Server will be continuously running

6. Server program will be waiting for smart phone to send accident coordinates.

7. When coordinates are received, using great Circle Distance formula, distance between Accident Location and the location of Emergency Service Providers stored in database is calculated.

8. Different lists are created according to distance.

9. First SMS will be sent to Emergency Service providers having least distance from accident location.

10. If message is not delivered to them, SMS will be sent to the number of Service Providers which are stored in another list

\section{Hardware:}

Hardware consists of Power Supply, microcontroller (IC AT 89s25), RS232 Module and Bluetooth module. In power supply, Bridge wave Rectifier is used with Transistor to generate positive voltage. Diode is used with capacitor to avoid reverse current of the circuit. Cristal Oscillator is used to generate digital signal, which is attached with micro controller. A103J is used with microcontroller to condense the circuit. Wireless Bluetooth serial trans-receiver module HC-06 is used for Bluetooth communication for serial communication IC MAX232 is used. Relay is used as sensor. Hardware should sense accident \& transfer bit to android smart phone via Bluetooth but due to compatibility issue Laptop's Bluetooth can be used to receive accident bit. Program in Laptop can be used to forward the same information to Smartphone via TCP/IP. The hardware can be classified into two parts such as Control Unit and Onboard Unit. 
CU:

The CU is responsible for sending accident event to the OBUs. CU sends only a true bit indicating the occurrence of accident. Control Unit receives input from sensors. But when accident is simulated using relay, $\mathrm{CU}$ will receive input when relay is fully compressed.

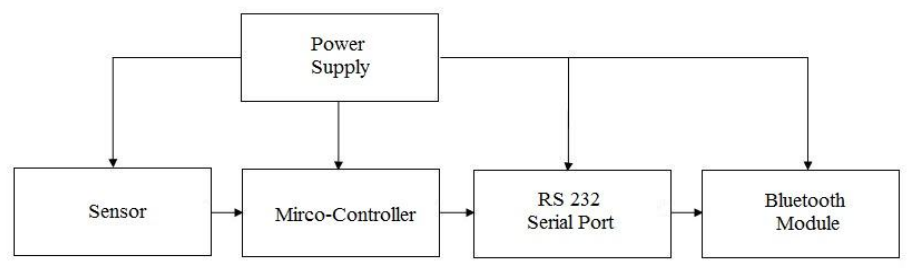

Figure 5: Control Unit

OBU:

In proposed system, Onboard Unit is a device, enriched with facilities of GPS and internet connectivity. This is a display device installed on dash board. The main objective of OBU is to obtain current location coordinates.

Onboard Application:

It is an Android application installed in OBU, which is responsible for receiving data from control unit. This application sends accident location information in terms of latitude and longitude to the server via internet

\section{Server:}

After receiving latitude and longitude from OBU, Server calculates the distance between accident location and location of Hospitals \& Police Station which is manually stored in database. Then server will send accident location information to nearest hospital, police \& relative via SMS.

For calculating the distance between accident location and assistance we use great circle distance formula mentioned below:

1. When two GPS coordinates are provided to the Great Circle Distance Formula, it will give the distance between the two locations in Kilometers or Nautical Miles.

2. The Great Circle Distance formula is as follows [6][7]:

Let $\phi_{1}, \lambda_{1}$ and $\phi_{2}, \lambda_{2}$ be the latitude and longitude of two points 1 and 2 , and $\Delta \phi, \Delta \lambda$ their absolute differences; then $\Delta \sigma$, the central angle between them is given by the spherical law of cosines: $\Delta \sigma=\arccos \left(\sin \phi_{1} \sin \phi_{2}+\cos \phi_{1} \cos \phi_{2} \cos \Delta \lambda\right)$.

The distance $d$, i.e. the arc length, for a sphere of radius $r$ and $\Delta \sigma$ given in

$d=r \Delta \sigma$.

Server application makes arrangement of providing latitude and longitude of emergency service provider from database to Great Circle Distance Formula. Distance is calculated from accident location to all these locations in database. It short lists the emergency service providers, whose distance is minimum from accident location.

\section{Web Application:}

It provides UI for Admin to add/update location information of Police Station \& Hospital and for Users to add/update location information of relatives and friends. Admin \& Users add the location of required people with the help of map.

\section{Sending SMS:}

There are various options available for implementing this module. Such as 1.Using GSM Module 2.Using SMS Gateway 3.Using java Supported Data Card 4.Using java supported phone. For making this project more cost efficient, using java supported Data Card could be the better option.

\section{View Map Application:}

It is an Android application which shows particular location on map whose GPS co-ordinates in terms of latitude \& longitude are given as input.

\section{CONCLUSION}

The purpose of this system is to detect accident and automate emergency assistance services. As a result, system is sending SMS to the nearest Emergency assistance service provider from accident location. Nearest Emergency assistance service provider is chosen according to their distance from accident location. Web 
application is used to add, modify the location of Emergency assistance service provider. Emergency assistance service providers are provided with an android application which shows a particular location on map when given co-ordinates of that particular location as an input. This will help them to find the exact accident location.

This paper presented a system, which allows detection of traffic accidents for improving the assistance to injured passengers by reducing the response time of emergency services through the efficient communication of relevant information about the accident. The proposed system requires installing CU \& OBU in the vehicles for detecting accidents and notifying it to the server, which will inform the nearest emergency service providers and victim's family members about the accident. The development of a low-cost prototype shows that it is feasible to incorporate this system in existing vehicles.

Like smart phones, in future cars will also get smarter. At that time, this system will play important role in making cars smarter. When India will have universal helpline (like 911 in USA), this system will work more efficiently.

This system can be used in following applications:-

1. Used as Fire Fighting System by using Smoke Detector Sensor.

2. Anti theft Application using combination of various sensors installed in Private properties (Banks, Offices).

3. Secure Home Application using combination of various sensors installed at doors, windows, roofs and lockers.

4. Personal Safety Application without using any external hardware.

5. Centralized Car Security System by installing Carbon Nano Tube (CNT) Sensors at Car windows.

Onboard Unit can replace all the present dashboard systems like A/C Controller, Music Systems, and Navigation System etc. OBU can also provide facilities like Calling, Skyping, voice controlling etc.

\section{Acknowledgements}

We take this opportunity to thank our project guide and Head of the Department Prof. Vaishali .S. Nandedkar for her valuable guidance in the completion of this project. We are also thankful to all the staff members of the Department of Information Technology of Padmabhooshan Vasantdada Patil Institute of Technology, Pune for their valuable time, support, comments, suggestions and persuasion. We would also like to thank the institute for providing the required facilities, Internet access and important books.

\section{REFERENCES}

[1] Manuel Fogue, Piedad Garrido, Francisco J. Martinez, Juan-Carlos Cano, Carlos T. Calafate, and Pietro Manzoni, “Assistance through Communication Technologies and Vehicles", IEEE VEHICULAR TECHNOLOGY MAGAZINE, SEPTEMBER 2012.

[2] Tanushree Dalai, "Emergency Alert and Service for Automotives for India", International Journal of Advanced Trends in Computer Science and Engineering (IJATCSE), Mysore, India, Vol.2, No.5, Pages: 08-12 (2013) Special Issue of ICETCSE 2013.

[3] R. Grzeszczyk, J. Merkisz, P. Bogus, and T.Kaminski "Methods and Procedures for testing the E- call in-vehicle unit for The purpose of its performance assessment and certification,"' in Proc. 21st Int. Technical Conf. Enhanced Safety of Vehicles (ESV), Stuttgart, Germany, June 2009, Paper 09-0332

[4] R. Bossom, R. Brignolo, T. Ernst, K. Evensen, A. Frotscher, W. Hofs, J. Jeaeaskeleainen, Z. Jeftic, P. Kompfner, T.Kosch, I. Kulp, A. Kung, A.-K. Mokaddem, A. Schalk, E. Uhlemann, and C. Wewetzer, "European ITS communication Architecture-Overall framework-Proof of concept implementation," EC FP7 Deliverable, EC Information Society Technologies Programme, Community Research and Development Information Service (CORDIS), European Union, Tech Rep., Mar 2009.

[5] Sunil Arya , David M Mount, Ruth Silverman , Angela Y.Wu, “An Optimal Algorithm for Approximate Nearest Neighbor Searching in Fixed Dimensions" in the Proceedings of the Fifth Annual ACM-SIAM Symposium on Discrete Algorithms, 1994.

[6] Earth:Great Circles, Geodesics, and Useful Projections",February 2006

[7] R. Bullock, "Great Circle Distances and Bearings between Two Locations", MDT, June 5, 2007. 